\title{
WEAK SEQUENTIAL DENSENESS IN BANACH SPACES
}

\author{
J. HOWARD
}

\begin{abstract}
H. P. Rosenthal [Some recent discoveries in the isomorphic theory of Banach spaces, Bull. Amer. Math. Soc. 84 (1978), 803-831] has shown that for separable Banach spaces, $X$ contains no subspace isomorphic to $l_{1}$ iff every bounded subset of $X$ is weakly sequentially dense in its weak closure (bwsd property). We show this is true for Banach spaces in general and compare the bwsd property to weak sequential denseness on arbitrary sets (weak $N$ sequential) and on relatively weakly compact sets (weak angelic).
\end{abstract}

We discuss three types of weak sequential denseness in a Banach space. They are defined as follows:

(1) $X$ is weak $N$-sequential [6, Problem 8.4.116] if $A$ is a subset of $X$ and $x \in \bar{A}^{w}$ (closure in the weak topology) imply there is a sequence $\left(a_{n}\right)$ in $A$ which converges weakly to $x$. From Problems 9.1.106 and 8.4.201 of [6] one can deduce that $X$ is weak $N$-sequential if and only if $X$ is finite dimensional.

(2) $X$ is bounded weak sequentially dense (bwsd) if every bounded subset of $X$ is weakly sequentially dense in its weak closure.

(3) $X$ is weak angelic if every relatively weakly compact subset of $X$ is weakly sequentially dense in its weak closure. It turns out that every Banach space has this property $[\mathbf{1}, \mathbf{3}]$.

Clearly, weak $N$-sequential $\Rightarrow$ bwsd $\Rightarrow$ weak angelic. Ryff [5] shows that $l_{1}$ does not have the bwsd property. Hence weak angelic does not imply bwsd. Our objective is to characterize the bwsd property.

THEOREM. The following are equivalent:

(a) Every bounded sequence in $X$ has a weak-Cauchy subsequence.

(b) $X$ has the bwsd property.

(c) Every separable subspace $Y$ of $X$ has the bwsd property.

(d) $X$ contains no subspace isomorphic to $l_{1}$.

ProOF. (a) $\Rightarrow$ (b) (This is an open question in [4].) Let $S$ be a bounded subset of $X$. Let $x^{\prime \prime} \in \overline{J S}^{w^{*}}$. By Whitley's method (see [1 and 2]) we construct a sequence $a_{1}, a_{2}, a_{3}, \ldots$ in $S$. By (a) there is at least one cluster point $F \in X^{\prime \prime}$ such that $F=x^{\prime \prime}$, in fact, $F=x^{\prime \prime}$ for each cluster point $F$ of $J a_{1}, J a_{2}, J a_{3}, \ldots$ Consequently, $J a_{1}, J a_{2}, J a_{3}, \ldots$ has a unique cluster point $F$. Since $\overline{J S}{ }^{w^{*}}$ is weak* compact and a sequence in a compact space with unique cluster point must converge to that point, $J a_{1}, J a_{2}, J a_{3}, \ldots$ converges weak ${ }^{*}$ to $F$. If $z$ is any element of $\bar{S}^{w}$, then $x^{\prime \prime}=J z$ is in $\overline{J S}^{w^{*}}$ and an application of what has been established yields a sequence $\left(a_{n}\right)$ in $S$ such that $\left(J a_{n}\right) \stackrel{w^{*}}{\longrightarrow} J z$. So $\left(a_{n}\right) \stackrel{w}{\longrightarrow} z$.

Received by the editors September 9, 1985 and, in revised form, January 22, 1986.

1980 Mathematics Subject Classification (1985 Revision). Primary 46B20; Secondary 46B22.

Key words and phrases. Weakly sequentially dense, weakly angelic. 
(b) $\Rightarrow$ (c) We show that bwsd is a hereditary property. To do this we show if there is a continuous one-to-one map from $Y$ into $X$ where $X$ is bwsd, then $Y$ is bwsd. Let $f: Y \stackrel{1-1}{\longrightarrow} X$ be continuous and $A$ be a bounded subset of $Y$ with $x \in \bar{A}^{w}$. Then $f$ is weak-weak continuous [2], so $f(x) \in f\left(\bar{A}^{w}\right) \subseteq \overline{f(A)}^{w}$. So there exists a sequence $f\left(a_{n}\right)$ such that $\left(f\left(a_{n}\right)\right) \stackrel{w}{\longrightarrow} f(x)$. Since $f$ is one-to-one, $f^{-1}$ is a closed map, so $\left(a_{n}\right) \stackrel{w}{\longrightarrow} x$.

(c) $\Rightarrow$ (d) By [5] $l_{1}$ does not have bwsd, and since bwsd is hereditary, $X$ cannot contain a subspace isomorphic to $l_{1}$.

(d) $\Rightarrow$ (a) This follows by Rosenthal's result [4].

From our characterization of bwsd, spaces such as $l_{2}$ and $c_{0}$ will have this property. Since they are not finite dimensional, they cannot be weak $N$-sequential. So there must exist in such spaces an unbounded set $A$ with a point in its weak closure that is not the limit of a sequence. Such a set for $l_{2}$ is $A=\left\{e_{m}+m e_{n}: 1<m<\right.$ $n<\infty\}$. Then $0 \in \bar{A}^{w}$, yet no sequence in $A$ weakly converges to 0 . Such a set for $c_{0}$ is $B=\left\{n \delta^{n}: n=1,2,3, \ldots\right\}$ (see Problem 8.4.120 of $[6]$ ).

\section{REFERENCES}

1. H. B. Cohen, Sequential denseness and the Eberlein-Smulian Theorem, Math. Ann. 172 (1967), 209-210.

2. R. Larsen, Functional analysis, Dekker, New York, 1973.

3. J. D. Pryce, A device of $R$. J. Whitley's applied to pointwise compactness in spaces of continuous functions, Proc. London Math. Soc. 23 (1971), 532-546.

4. H. P. Rosenthal, Some recent discoveries in the isomorphic theory of Banach spaces, Bull. Amer. Math. Soc. 84 (1978), 803-831.

5. J. V. Ryff, The weak closure of a certain set in $l_{1}$, Amer. Math. Monthly 81 (1974), 69-70.

6. A. Wilansky, Modern methods in topological vector spaces, McGraw-Hill, New York, 1978.

Division of Science and Mathematics, New Mexico highlands University, LAS VEGAS, NEW MEXICO 87701 